\title{
Early outcomes of cleft and palatal width following anterior palate repair (vomerine flap) in infants with wide cleft lip and palate
}

\author{
Arman Zaharil Mat Saad ${ }^{1,2,3}$, Koh Siang Chai ${ }^{1,3}$, Wan Azman Wan Sulaiman ${ }^{1,3}$, \\ Siti Fatimah Noor Mat Johar ${ }^{1,3}$, Ahmad Sukari Halim ${ }^{1,3}$ \\ ${ }^{1}$ Reconstructive Sciences Unit, School of Medical Sciences, Universiti Sains Malaysia, Kota Bharu; ${ }^{2}$ Plastic and Reconstructive Surgery Unit, \\ MSU Medical Centre, Management and Science University, Shah Alam; ${ }^{3}$ Hospital Universiti Sains Malaysia, Health Campus, Universiti \\ Sains Malaysia, Kota Bharu, Malaysia
}

Background Anterior palatal repair performed during cleft lip repair using a vomerine flap may assist in recruiting additional soft tissue for subsequent completion of palatoplasty, especially in patients with a wide cleft. We present our early results in the hope of triggering a re-evaluation of this technique regarding its advantages for maxillary growth through further studies of patients with a wide cleft.

Methods A retrospective analysis of patients with complete unilateral and bilateral cleft lip and palate was performed, including cleft and palatal measurements taken during initial surgery (lip repair together with anterior palate repair) and upon completion of palatoplasty. Results In total, 14 patients were included in this study, of whom nine (63.3\%) had unilateral cleft lip and palate and five (37.5\%) had bilateral cleft. All patients had a wide cleft palate. Lip and anterior palate repair was done at a median age of 3 months, while completion of palatoplasty was done at a median age of 10.5 months. Measurements taken upon completion of palatoplasty showed significant cleft width reduction in the mid-palate and intertubercle regions; however, the palatal arch distances at nearby landmarks showed non-significant marginal changes.

Conclusions Anterior palate repair using a vomerine flap significantly reduced the remaining cleft width, while the palatal width remained. Further research is warranted to explore the long-term effects of this technique in wide cleft patients in terms of facial growth.

Keywords Cleft lip / Cleft palate / Vomerine flap / Wide

\author{
Correspondence: \\ Arman Zaharil Mat Saad \\ Plastic and Reconstructive Surgery \\ Unit, MSU Medical Centre, \\ Management and Science University, \\ Jalan Boling Padang 13/64, Seksyen \\ 13, Shah Alam 40100, Malaysia \\ Tel: +60-3-5526-2888 \\ Fax: +60-3-5524-3656 \\ E-mail: armanzaharil@gmail.com
}

Received: February 20, 2019 • Revised: August 21, 2019 • Accepted: September 24, 2019

pISSN: 2234-6163 • elSSN: 2234-6171 • https://doi.org/10.5999/aps.2019.00227• Arch Plast Surg 2019;46:518-524

\section{INTRODUCTION}

Cleft lip and palate is one of the most common congenital anomalies, affecting approximately 1 in 750 to 1,000 live births worldwide. Due to the complexity of the anatomical and functional abnormalities involved, various cleft centers have developed their own treatment protocols in terms of the timing of surgery, surgical techniques, endorsement of a multidisciplinary 
approach, and schedule of follow-up. All these efforts aim to restore velopharyngeal function for normal speech production, as well as a separate oral and nasal cavity, without causing significant disturbances to maxillary growth [1]. Unfortunately, no single surgical technique or protocol has satisfied all these conditions.

The benefits of one-stage palatoplasty and two-stage palatoplasty are topics of ongoing debate. One-stage palatoplasty comprises two-flap palatoplasty with intravelar veloplasty, such as Bardach two-flap technique and Von Langenbeck technique with a lateral relaxing incision. This technique allows the restoration of functional and competent velopharyngeal function, as well as closure of the palatal cleft, in a single operation. Surgeons who adopt two-stage palatoplasty repair the secondary palate first, leaving the remaining palatal defect to be repaired at a later age. Repairing the secondary palate at an early age allows normal speech development, while avoiding extensive mucoperiosteum dissection for hard palate closure, which hinders maxillary and midface growth [1-3].

Bardach [4] defined a width of $1.5 \mathrm{~cm}$ as indicative of a wide cleft palate [5]. Bumsted [6] defined an extremely wide cleft palate to be present in cases where "the width of the cleft defect is larger than the sum of the combined widths of the remaining palate." Palatoplasty in cases of wide cleft palate remains a surgical challenge due to the high risk of oronasal fistula formation as a result of tension closure of the nasal mucosal layer. Even if a unilateral or bilateral vomerine flap is utilized to facilitate the closure of the nasal layer, there is still a high risk of fistula formation over the posterior limit of the septum due to dehiscence as a result of tension. If the nasal layer at the posterior limit of the septum is left to heal with secondary intention, scarring will cause the reconstructed velum to migrate anteriorly, resulting in velopharyngeal insufficiency [7].

In an attempt to overcome these difficulties and resolve the controversy surrounding mid-facial growth issues, the first author repaired the anterior part of the palate at the time of lip repair surgery.

\section{METHODS}

A retrospective study of all patients with complete unilateral and bilateral cleft lip and palate who underwent lip repair with anterior palate repair and subsequent palatoplasty from 2013 to 2017 (a 5-year period), were included in this study. The patients were identified through an electronic operative database; subsequently, medical records and photo documentation were retrieved. Relevant data was collected and statistical analyses were performed. Patients with missing medical records and incom- plete data were excluded from this study. This study was exempted by our Institutional Review Board from Ethical Review (USM/JEPeM/18100481).

\section{Anthropometric measurements}

The intertubercle cleft width, mid-palatal cleft width, intertubercle distance, mid-palatal distance, and cleft length were measured intraoperatively before the incision was made (Figs. 1, 2). Anthropometric measurements of the cleft lip, nose, and palate were routinely measured intraoperatively as a part of operative documentation, making this study possible. The intertubercle distance was defined as the distance between the most posterior parts of the maxillary alveolar arch on the left and right, while the intertubercle cleft width was defined the width of the palatal cleft measured at this level. The mid-palatal distance was defined as the width of the maxillary alveolar arch at the level of the mid-hard palate, while the mid-palatal cleft width was the width of the cleft palate at this level. The cleft length was the length of the cleft palate, measuring from the alveolus to the posterior extent of the soft palatal cleft.

\section{Surgical techniques}

The surgical techniques that were utilized were similar to those described by Pichler and popularized by the Oslo cleft lip and palate team in Norway $[8,9]$. Operations were performed on both unilateral and bilateral cleft lip and palate patients. Anterior

\section{Fig. 1. Schematic diagram of measurements}

t-t', intertubercle distance; i-i', intertubercle cleft width; $p-p^{\prime}$, midpalatal distance; $m-m$ ', mid-palatal cleft width, where both " $x$ " values indicate an equal distance; $a-L$, cleft length.

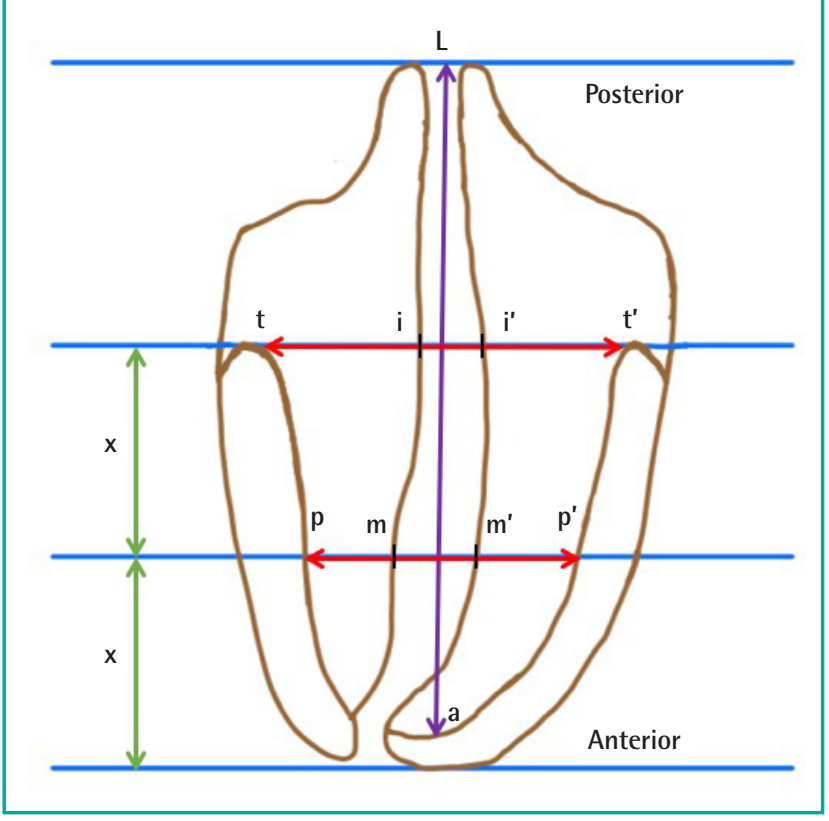


palate repair was performed at the time of lip repair surgery at the age of 3 months. No presurgical orthopedics were used for patients with unilateral cleft prior to surgery, but they were used in selected patients with bilateral cleft with a very prominent premaxilla. Patients' guardians provided informed consent and the procedure was carried out under general anesthesia.

For patients with unilateral cleft lip and palate, the modified Millard rotation-advancement technique was used for lip repair, while the modified Millard technique was used in cases with bilateral cleft [10-12]. In cases of unilateral complete cleft, the lip repair incision and dissection were first done using modified Millard rotational advancement flaps. The incision was extended at the cleft edge around the gingiva and alveolus (to facilitate complete soft tissue closure), around the gingivo-periosteal flaps, until the junction of the oral mucoperiosteal and nasal layers of the cleft side of the hard palate and the junction between the oral mucoperiosteal and vomerine mucoperiosteal junction on the non-cleft side. At this stage, lip closure was deferred until completion of the dissection and closure of the anterior palate, allowing unrestricted access for the intra-oral component of surgery. A vomerine flap was raised and reflected to the under-surface of the oral mucoperiosteal flap, which was raised along the cleft edges, overlapped, and sutured in a double-breasted manner (Fig. 3). The length of the repair at the anterior palate depended on the reach of the vomerine flap. The oral mucoperiosteal flap on the cleft side was raised posteriorly until this limit, or until the junction between the hard and soft palate, if the reach of the vomerine flap was not an issue. All approximations were done using absorbable sutures (a combination of mattress $4 / 0$ Vicryl [Ethicon Inc., Somerville, NJ, USA] on the $5 / 8$ circle needle and simple interrupted 5/0 Polydioxanone [Ethicon Inc.]). Upon completion of the anterior palatal suturing, lip re-

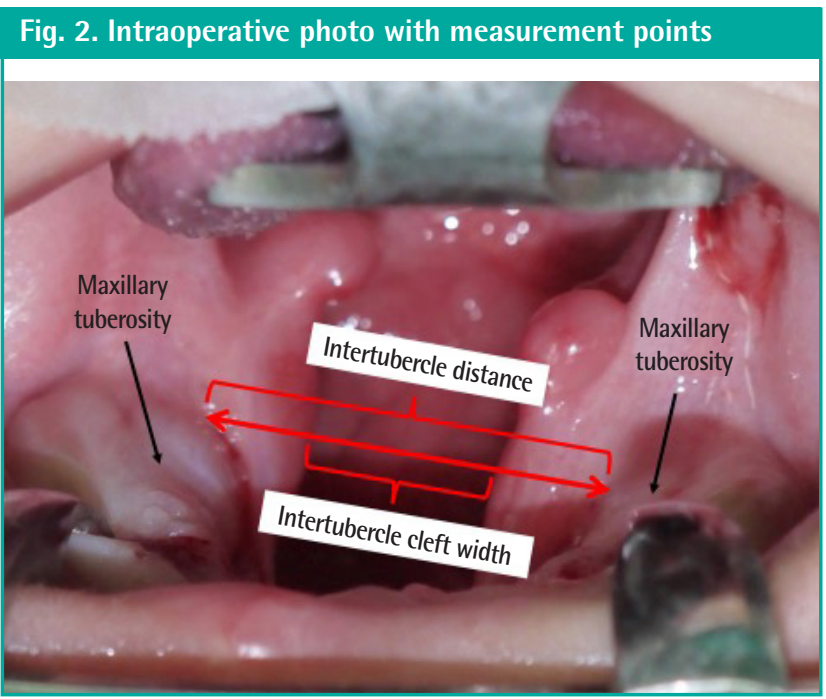

pair was resumed with closure in layers. The raw area after anterior palatal repair was left to granulate and to heal by secondary intention.

In bilateral complete clefts, the same sequence was followed. Once the lip incision and dissection were completed, the anterior palate was closed. Priority was given to the wider cleft side. The incision for the vomerine flap was made slightly off-center towards the narrower cleft side to facilitate extra reach on the wider cleft side. It is recommended to perform dissection on only one side of the vomerine flap to avoid disruption of blood supply to the premaxilla. However, when the cleft gaps are of unequal size, or one of the clefts is narrow or sometimes touches the lateral segment (as in the majority of our cases), the vomerine flap on the narrower cleft was carefully and minimally raised to prevent devascularization of the premaxilla; therefore, bilateral anterior palatal repair could be achieved (Fig. 4). Other details were as described above for unilateral cleft repair.

The second operation was performed for complete closure of the palate. It was planned between the ages of 9 and 12 months using Bardach's two-flap technique together with intravelar veloplasty [13]. At this stage, the cleft palate resembled a cleft secondary palate, due to closure of the anterior palatal cleft at the time of lip repair (Fig. 5). Similar measurements were made intraoperatively prior to the incision.

\section{Statistical analysis}

The power of the study was 0.8 , with a level of significance of 0.05 , to detect a difference in intertubercle distance, intertubercle cleft width, mid-palatal distance, mid-palatal cleft width, and

\section{Fig. 3. Closure using a reflected vomerine flap}

A single-layer closure using a reflected vomerine flap that was attached to the under-surface of the oral mucoperiosteal flap raised along the cleft edge.

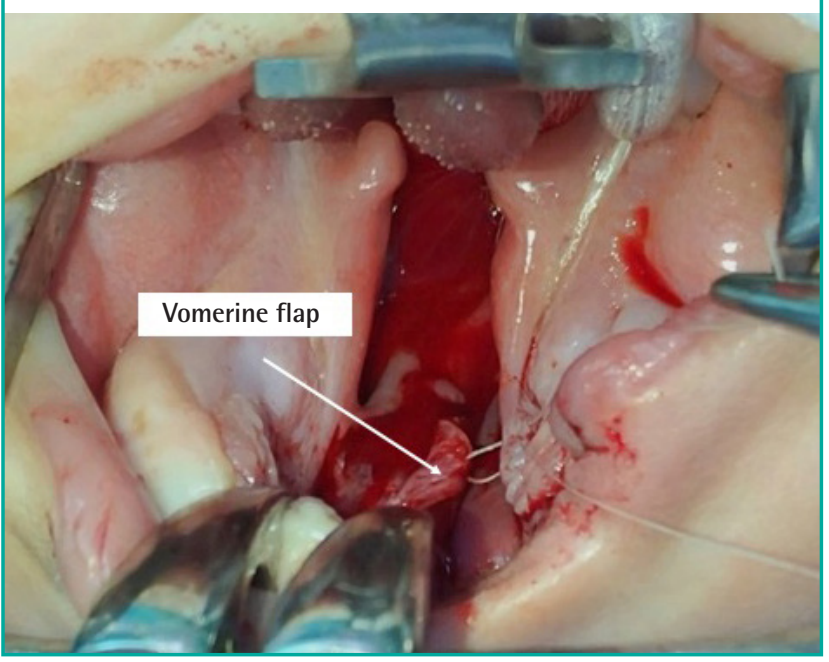




\section{Fig. 4. Complete bilateral cleft lip and palate}

$\mathrm{R}$ and $\mathrm{L}$ denote the patient's right and left side, respectively. (A) The cleft palate on the right side (almost touching) is narrower than the left side (the photo is upside-down, corresponding to the surgeon's intraoperative view). (B) Bilateral vomerine flaps were raised, sutured to the left oral mucoperiosteal flap (white arrow), and partially sutured on the right (red arrow). The denuded vomer is marked by a yellow arrow. (C) Photograph taken 9 months after lip repair and anterior palatal repair show a well-healed and mucosalized vomerine flap, as well as healthy and good growth of the premaxilla with tooth eruption.
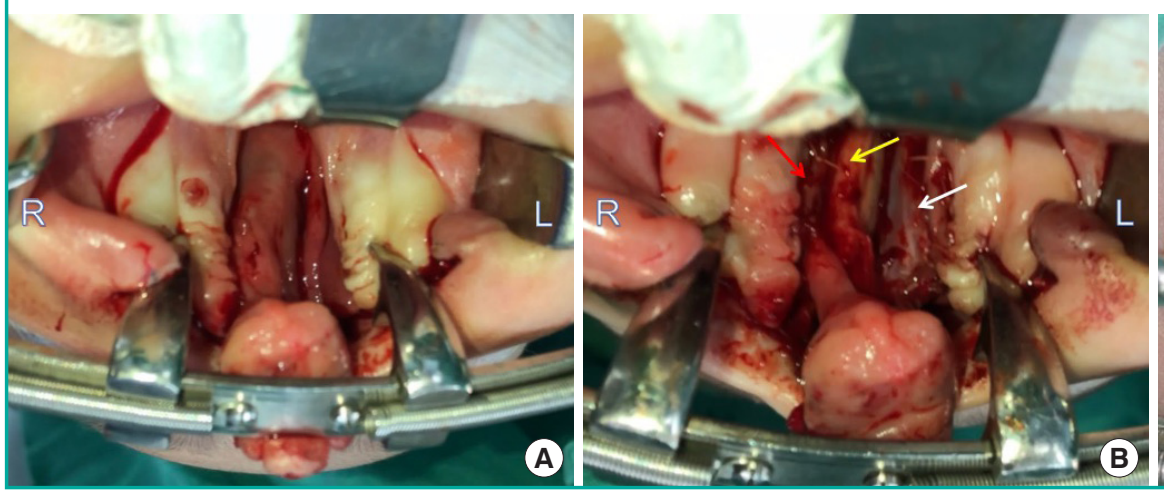

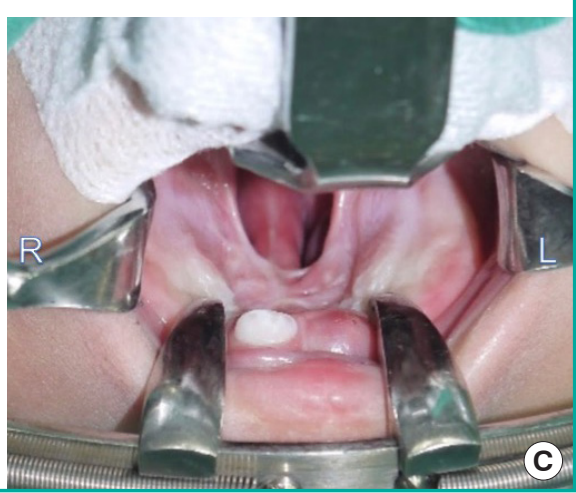

\section{Fig. 5. Intraoperative photo during completion of palatoplasty}

Intraoperative photo during palatoplasty in a 1-year-old. Lip and anterior palate repair had been done previously.

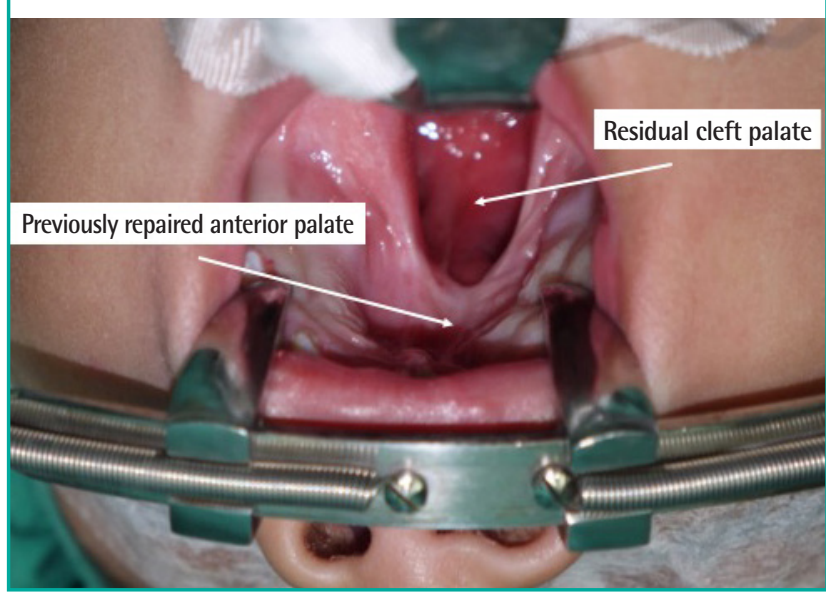

cleft length between the two procedures. The Wilcoxon signedrank test was performed to test the significance of differences in these variables between the time of lip repair and completion of palatoplasty. SPSS statistical software version 24 (IBM Corp., Armonk, NY, USA) was used.

\section{RESULTS}

Of the 23 patients who had cleft lip with anterior palatal repair, a total of 14 patients were included in this study. Nine patients were excluded due to missing medical records and/or incomplete documentation of measurements. Ten patients (71.43\%) were male, while four patients $(28.57 \%)$ were female. Nine patients $(64.29 \%)$ had unilateral cleft lip and palate, while the other five patients (35.71\%) had bilateral cleft. The median age was
Table 1. Wide cleft palate according to Bardach's and Bumsted's definitions

\begin{tabular}{|ccccc|}
\hline $\begin{array}{c}\text { Case } \\
\text { no. }\end{array}$ & $\begin{array}{c}\text { Cleft } \\
\text { width } \\
(\mathbf{m m})\end{array}$ & $\begin{array}{c}\text { Sum of the combined } \\
\text { widths of the remaining } \\
\text { palate }(\mathbf{m m})\end{array}$ & Bardach $^{\text {a) }}$ & Bumsted ${ }^{\text {b) }}$ \\
\hline 1 & 18 & 12 & Yes & Yes \\
2 & 19 & 16 & Yes & Yes \\
3 & 16 & 20 & Yes & Yo \\
4 & 16 & 15 & Yes & Yes \\
5 & 19 & 17 & Yes & Yes \\
6 & 15 & 15 & Yes & Yes \\
7 & 12 & 10 & Yes & Yes \\
8 & 18 & 16 & Yes & Yes \\
9 & 16 & 22 & Yes & No \\
10 & 19 & 14 & Yes & Yes \\
11 & 15 & 17 & Yes & Yes \\
12 & 18 & 14 & 13 (92.86) & 11 (78.57) \\
\hline 13 & 15 & 14 & & \\
14 & 14 & 13 & & \\
\hline
\end{tabular}

3 months (interquartile range [IQR], 0 months) at lip and anterior palate repair surgery, with a median weight of $5 \mathrm{~kg}(\mathrm{IQR}, 1.6$ months). Thirteen of the 14 patients (92.86\%) had wide cleft palate according to Bardach's definition $[4,5]$. Eleven patients (78.57\%) had extremely wide cleft palate according to definition of Bumsted (Table 1) [6].

Only one patient (7.14\%) had bleeding from the anterior palate postoperatively, which stopped spontaneously without needing exploration for hemostasis in the operating theater. All patients were discharged 2 days postoperatively. The second operation to repair the remaining palatal defect (i.e., completion of 
Table 2. Comparison of the variables measured at the time of lip repair and anterior palatal repair with those obtained upon the completion of palatoplasty using the Wilcoxon signed-rank test

\begin{tabular}{|lccrr|}
\hline Variable & Lip repair and anterior palatal repair & Completion of palatoplasty & Z statistic & P-value \\
\hline Intertubercle distance & $31.50(35.25-29.75)$ & $31.50(34.50-27.00)$ & -0.24 & 0.81 \\
Intertubercle cleft width & $17.00(18.25-14.75)$ & $10.00(12.00-8.00)$ & -3.30 & $<0.01$ \\
Mid-palatal distance & $28.00(30.50-23.00)$ & $26.00(28.00-24.00)$ & -0.60 & 0.55 \\
Mid-palatal cleft width & $15.50(18.25-13.75)$ & $0.00(6.50-0.00)$ & -3.30 & $<0.01$ \\
Cleft length & $44.50(45.00-35.00)$ & $26.50(29.75-22.00)$ & -2.80 & $<0.01$ \\
\hline
\end{tabular}

Values are presented as median (interquartile range). All unit of data is millimeter.

palatoplasty and intravelar veloplasty) was performed at a median age of 10.5 months (IQR, 4 months). Median weight at the time of surgery was $8 \mathrm{~kg}(\mathrm{IQR}, 2.2 \mathrm{~kg})$. After palatoplasty, all patients were discharged on postoperative day 2.

Measurements taken during both procedures showed a significant reduction in intertubercle cleft width, mid-palatal cleft width, and cleft length. The median intertubercle cleft width decreased from $17.0 \mathrm{~mm}$ to $10.0 \mathrm{~mm}(\mathrm{P}<0.01)$, while the median mid-palatal cleft width decreased from $15.5 \mathrm{~mm}$ to $0.0 \mathrm{~mm}$ $(\mathrm{P}<0.01)$. The median cleft length decreased from $44.5 \mathrm{~mm}$ to $26.5 \mathrm{~mm}(\mathrm{P}<0.01)$. Changes in the intertubercle distance and mid-palatal distance were insignificant (Table 2).

None of the patients had developed palatal fistula at a clinical follow-up 2 months postoperatively or during subsequent follow-up over a 1-year period; the longest follow-up was 5 years. The patients with bilateral cleft $(n=5)$ showed no signs of maxillary atrophy or necrosis during follow-up (Fig. 4).

\section{DISCUSSION}

The effects of surgical correction of lip and palatal clefts remain a controversial issue. Many surgical correction protocols are available, but there is a lack of a gold-standard protocol for palatal closure. Another issue relates to maxillary growth $[1,2]$. In this study, we evaluated the early effect of incorporating anterior palatal closure using a vomerine flap as a single-layer closure during lip repair. This technique has been used by the first author since 2013, and we evaluated the early outcomes of this technique for cleft width, cleft length, and maxillary arch dimensions measured at the time of lip repair performed around 3 months of age and subsequent repair of the palate about 6 to 9 months later.

It is interesting to note that all the patients in this cohort had a wide cleft palate (Table 1). According to Bardach's definition, $92.86 \%$ of our patients had a cleft width more than $1.5 \mathrm{~cm}$, and when using the definition of Bumsted, 78.57\% of patients had a cleft width wider than the tissue available on the palate lateral to the cleft (sum of the widths of the left and right oral mucoperi- osteal flaps) [4-6]. As shown in Table 2, the initial median cleft width in the intertubercle and mid-palate regions was $17.00 \mathrm{~mm}$ and $15.50 \mathrm{~mm}$, respectively. In patients with a wide cleft, native tissue on the palate region is scarce; therefore, many surgeons will accept an anterior oronasal fistula in favor of speech by pushing the tissue posteriorly to aid velopharyngeal closure for speech. Closure of wide cleft palate is a significant challenge due to the relatively small amount of tissue available to close a large defect without tension. This can cause impaired palatal wound healing and the subsequent development of oronasal fistula. Other factors that can contribute to fistula formation include bleeding as a result of extensive dissection and infection. The incidence of oronasal fistula after palatoplasty was reported to be between $12 \%$ and $23 \%$ [1]. Here, we would like to acknowledge that one of the advantages of anterior palatal repair in this series was the $0 \%$ incidence of fistula. None of the patients with bilateral cleft developed atrophy or necrosis of the premaxilla due to disruption of the blood supply, even though bilateral vomerine flaps were raised in these cases. Despite these good outcomes, we still advise caution and careful judgement on whether it is safe to proceed with bilateral dissection.

This procedure also yielded a significant reduction of cleft width in the mid-palatal region and the intertubercle region, with $\mathrm{P}<0.001$ for both parameters. In the intertubercle region, the median cleft width decreased from $17.00 \mathrm{~mm}$ to $10.00 \mathrm{~mm}$ by the time of palatoplasty. In the mid-palatal region, the median cleft width showed an even greater reduction to $0 \mathrm{~mm}$ from $15.50 \mathrm{~mm}$. This reduction could have been due solely to the newly formed tissue recruited from the vomerine flap procedure for closure of the anterior palate at the time of lip surgery. Alternatively, it could have been due to pulling of the palatal arches medially over time after lip surgery, or potentially both factors. In this early study, the authors showed that the median mid-palatal distance (width) and inter-tubercular distance (width) were marginally reduced and remained unchanged, respectively. The median mid-palatal distance decreased from $28.00 \mathrm{~mm}$ to 26.00 $\mathrm{mm}$ from the time of lip repair to palatoplasty. However, the median intertubercle distance remained $31.50 \mathrm{~mm}$ over a 
6-month period. This might have been due to the correction of unopposed outward growth of the palatal shelves during the early life of the patient. In a study published by Maggiulli et al. [9] in 2014, anterior and posterior arch width was described in two groups of patients, who did or did not receive a vomerine flap for anterior palatal closure. They also found that the anterior arch had a reduction in width, but in contrast to our findings, the posterior arch showed a minimally increased width between the two procedures, which were performed only 3 months apart (compared to our study, where the interval was 6-9 months apart). A more recent paper from the same center published in 2018 reporting 10-year follow-up outcomes with cephalometric data on maxillary growth concluded that performing a vomerine flap during lip repair surgery did not affect maxillary growth in comparison to the control non-vomerine repair cohort [14]. Our findings may raise an alarm, alerting clinicians and researchers to the possibility of early growth restrictions following anterior palatal repair. However, our patients represent a different cohort of cases, with wide clefts; therefore, a long-term study and follow-up on maxillary growth are required for the patients in our series, as they are different from those analyzed in previous studies [14,15].

Even though performing anterior palatal repair at the time of lip repair slightly prolonged the time of the first operation, the cleft width narrowed by the time we completed palatoplasty as the child grew. A narrower palatal cleft gap made subsequent completion of palatoplasty less challenging, as the surgeon had more available soft tissue to be recruited and less movement of the flaps was required to achieve closure with minimal tension, which decreased the risk of oronasal fistula formation. Compared to two-stage palatoplasty, which may require multiple surgical procedures to repair both the lip and palate, our technique has the advantage of being a single-stage variation of palatoplasty that only requires two operations. In patients with no underlying comorbidities, lip and palate repair is usually complete at 1 year of age. A study showed that the use of a single-layer vomerine flap at the time of lip repair reduces the risk of symptomatic fistula formation, providing a good foundation for a mixed dentition alveolar bone graft, as well as an acceptable arch form [1416]. Our technique allows the complete closure of anterior oronasal fistulas, while recruiting additional tissue for subsequent completion of palatoplasty. This permits intravelar veloplasty and push-back of the mucoperiosteal flaps to be performed during the second operation to reduce the risk of developing velopharyngeal insufficiency, with no need to worry about cleft gap or fistula at the anterior palate. Our results showed a $0 \%$ fistula rate and complete closure of the anterior cleft gap in all cases. However, as the anterior palate repair requires the raw area to heal by secondary intention, fibrosis, and scarring, there might be some difficulty in elevating mucoperiosteal flaps during the second stage of surgery (completion of palatoplasty). This additional scarring in wide cleft palate patients may affect mid-facial growth differently from the patterns reported in previous studies $[14,15]$, and we are still currently following our patients to observe the effects on maxillary growth.

We only observed one patient with postoperative bleeding from the anterior palate, which resolved spontaneously without the need for hemostasis in the operating theater. Clinical followup after palatoplasty revealed that none of the patients had developed palatal fistula.

This study revealed a significant reduction in cleft width, both in the mid-palatal region and in the intertubercle regions. In addition, the palatal arch width in the mid-palate and intertubercle areas did not significantly differ between the two operations, which warrants a long-term study to determine the effect on maxillary growth in our cohort of patients with wide palatal cleft. We also acknowledge that further study is required to determine the real net effect of anterior palate closure using a vomerine flap through a comparison with a control group of patients with lip repair alone and in normal children of a similar age group to determine the normal dimensions of palatal width.

\section{NOTES}

\section{Conflict of interest}

No potential conflict of interest relevant to this article was reported.

\section{Ethical approval}

The study was approved by the Institutional Review Board of Universiti Sains Malaysia, Health Campus (IRB No. USM/JE$\mathrm{PeM} / 18100481$ ) and performed in accordance with the principles of the Declaration of Helsinki. Informed consent for the study was obtained from the patient's parent.

\section{Patient consent}

The patient's parent provided written informed consent for the publication and the use of the images.

\section{Author contribution}

Conceptualization: Mat Saad AZ, Wan Sulaiman WA, Halim AS. Data curation: Chai KS, Mat Johar SFN, Mat Saad AZ. Formal analysis: Chai KS, Mat Saad AZ. Methodology: Mat Saad AZ, Wan Sulaiman WA, Halim AS, Chai KS, Mat Johar SFN. Project administration: Mat Saad AZ, Wan Sulaiman WA, Halim AS. Visualization: Mat Saad AZ, Chai KS, Mat Johar 
SFN. Writing- original draft: Mat Saad AZ, Chai KS. Writingreview \& editing: Mat Saad AZ, Wan Sulaiman WA, Halim AS. Approval of final manuscript: all authors.

\section{ORCID}

Arman Zaharil Mat Saad https://orcid.org/0000-0002-40036783

Koh Siang Chai https://orcid.org/0000-0001-9787-4510

Wan Azman Wan Sulaiman https://orcid.org/0000-00020600-9765

Siti Fatimah Noor Mat Johar https://orcid.org/0000-00034120-4918

Ahmad Sukari Halim https://orcid.org/0000-0001-89996403

\section{REFERENCES}

1. Lin KY, Black JS, Wang JS, et al. An outcome study of a 2-flap pushback palatoplasty used in the treatment of wide cleft palates. J Craniofac Surg 2015;26:620-4.

2. Friede H. Maxillary growth controversies after two-stage palatal repair with delayed hard palate closure in unilateral cleft lip and palate patients: perspectives from literature and personal experience. Cleft Palate Craniofac J 2007;44:129-36.

3. Liau JY, Sadove AM, van Aalst JA. An evidence-based approach to cleft palate repair. Plast Reconstr Surg 2010;126: 2216-21.

4. Bardach J. Book Salyer and Bardach's atlas of craniofacial and cleft surgery. Vol. II: Cleft lip and palate surgery. Philadelphia: Lippincott-Raven; 1999.

5. Aboul-Wafa AM. Islandized mucoperiosteal flaps: a versatile technique for closure of a wide palatal cleft. Can J Plast Surg 2012;20:173-7.
6. Bumsted RM. A new method for achieving complete twolayer closure of a massive palatal cleft. Arch Otolaryngol 1982;108:147-50.

7. Khan K, Hardwicke J, Seselgyte R, et al. Use of the sphenoid flap in repair of the wide cleft palate. Cleft Palate Craniofac J 2018;55:437-41.

8. Abyholm FE, Borchgrevink HC, Eskeland G. Cleft lip and palate in Norway. III. Surgical treatment of CLP patients in Oslo 1954-75. Scand J Plast Reconstr Surg 1981;15:15-28.

9. Maggiulli F, Hay N, Mars M, et al. Early effect of vomerine flap closure of the hard palate at the time of lip repair on the alveolar gap and other maxillary dimensions. Cleft Palate Craniofac J 2014;51:43-8.

10. Millard DR Jr. Rotation-advancement principle in cleft lip closure. Cleft Palate J 1964;12:246-52.

11. Millard DR. Bilateral cleft lip and a primary forked flap: a preliminary report. Plast Reconstr Surg 1967;39:59-65.

12. Millard DR Jr. Refinements in rotation-advancement cleft lip technique. Plast Reconstr Surg 1964;33:26-38.

13. Bardach J. Two-flap palatoplasty: Bardach's technique. Oper Tech Plast Reconstr Surg 1995;2:211-4.

14. Hay N, Patel B, Haria P, et al. Maxillary growth in cleft lip and palate patients, with and without vomerine flap closure of the hard palate at the time of lip repair: a retrospective analysis of prospectively collected nonrandomized data, with 10-year cephalometric outcomes. Cleft Palate Craniofac J 2018;55: 1205-10.

15. Semb G. A study of facial growth in patients with unilateral cleft lip and palate treated by the Oslo CLP Team. Cleft Palate Craniofac J 1991;28:1-21.

16. Bergland $\mathrm{O}$. Changes in cleft palate malocclusion after the introduction of improved surgery. Rep Congr Eur Orthod Soc 1967:383-97. 